\title{
Perceptual Quality Metrics for 3D Meshes: Towards an Optimal Multi-Attribute Computational Model
}

\author{
Guillaume Lavoué \\ Université de Lyon, CNRS \\ Insa-Lyon, LIRIS UMR 5205 \\ France
}

\author{
Irene Cheng, Anup Basu \\ Department of Computing Science \\ University of Alberta \\ Canada
}

\begin{abstract}
D graphical data, commonly represented using triangular meshes, are deployed in a wide range of application processes including compression, filtering, watermarking, and simplification. These processes often introduce geometric distortions which affect the visual quality of the ultimate data visualization. In order to accurately evaluate perceptual impacts caused by the distortions, assessment metrics on 3D Mesh Visual Quality (MVQ) have been extensively discussed in the literature. Researchers recommended various metrics to predict the adverse effects that visual artifacts can have in applications. Most of these metrics are based on geometric attributes, conventional geometric distance, Laplacian coordinates, different types of curvature computation, and dihedral angles. We hypothesize that an optimal combination of multiple attributes associated with a 3D mesh surface can contribute to better perceptual prediction than single attributes used separately. In this paper, we use two user studies to validate our hypothesis. Our contributions are: (1) providing a detailed analysis of the most relevant geometric attributes for mesh quality assessment, and (2) introducing a new perceptual evaluation metric based on multiple attributes, with the optimal combination determined through machine learning techniques. Statistical quantitative analysis shows that our metric delivers better results than other state-of-the-art approaches. The proposed method is simple to implement and fast in execution. Moreover, our framework can easily be expanded to accommodate additional surface attributes.
\end{abstract}

Index Terms-Visual quality assessment, Perceptual metric, 3D Mesh, Geometric attributes, Machine learning.

\section{INTRODUCTION}

Three-dimensional mesh data are commonplace in many application domains (scientific visualization, digital entertainment, mechanical engineering and cultural heritage). 3D mesh visualization capability has expanded from desktops to mobile devices such as tablets and smartphones. Furthermore, the visualization of 3D web content is also possible. Application demands lead to a wide range of 3D mesh processes which include transmission, compression, simplification, retargeting, filtering, watermarking and so on. These operations inevitably introduce distortions which often alter the visual quality of the rendered data. In order to deliver satisfactory Quality of Experience to application users, 3D Mesh Visual Quality (MVQ) metrics have been intensively studied in the literature [1], [2]. The objective is to predict the visual quality of the distorted 3D data in compared to the 978-1-4799-0652-9/13/\$31.00 (C)2013 IEEE original.

Many MVQ metrics are inherited from Image Quality Assessment (IQA) metrics [3], which were designed for assessing the quality performance of image processing algorithms, in particular during the compression and transmission process. Some of the evaluation concepts have been applied in Mesh Visual Quality metrics (see section II). Most commonly used 3D mesh surface attributes in MVQ include geometric distances, Laplacian coordinates, curvature computations, and dihedral angles. Local and global considerations, as well as appropriate weightings are usually incorporated in order to make the assessment consistent with how the Human Visual System (HVS) perceives 3D objects. It is realized by researchers that simple geometric measures (like Hausdorff distance or mean squared error) do not correlate well with human opinion; thus, perceptual factors need to be integrated into the metrics. However, there is a lack of consensus on which 3D mesh surface attributes have perceptually significant impact on visual quality.

This paper proposes a multiple feature metric where parameters are optimized using machine learning techniques. Our contributions include: (1) evaluating, based on Mean Opinion Score (MOS) data derived from two subjective quality assessment experiments, the relative effectiveness of eight attributes commonly used to predict visual qualities in MVQ metrics ; and (2) proposing a new quality assessment metric using perceptually significant 3D mesh surface attributes. The proposed metric not only has fast execution time but is also simple to implement. It provides results better than other state-of-the-art approaches.

The rest of this paper is organized as follows: Section II discusses related work on IQM and 3D MVQ assessments; Section III presents the commonly used 3D mesh attributes for predicting visual quality; Section IV provides information on the subjective mean opinion score databases used in our evaluation experiments; and finally Section V describes the evaluation protocols, the proposed metric and experimental results. 


\section{RELATED WORK}

Perceptual factors play an important role in research on computer graphics [4], which can significantly affect the quality performance of many operations, rendering and simplification. Simple image quality metrics rely on pixelto-pixel differences of the luminance attribute, PSNR and Root Mean Square error, while more complex metrics take advantage of the human visual system characteristics, such as the contrast sensitivity function, multi-channel decomposition, luminance and contrast masking. Many 3D mesh quality evaluation concepts consider directly 2D image metrics (RMS error, SSIM [5], Sarnoff VDM [6], Daly VDP [7]). For instance, Lindstrom and Turk [8] and more recently Qu and Meyer [9] guide their simplification algorithms using 2D perceptual models. For rendering, 2D perceptual metrics are used to determine the desired precision based on the observer's viewpoint, the best level-of-detail [10], or the best ray sampling density [11]. However, as recently reported by Cadik et al. [12], synthetic images coming from computer graphics rendering are prone to specific artifacts which are not correctly predicted by standard image quality metrics. Therefore, new perceptual criteria were introduced to address this insufficiency [13]-[15].

All the previously mentioned works concern 2D image metrics. However, as observed in the subjective experiments of Rogowitz and Rushmeier [16], the perceived quality of 3D objects may not be correctly predicted based on the quality of their $2 \mathrm{D}$ projections due to the additional depth perception. Therefore, several authors introduced perceptually-motivated metrics operating directly on the mesh geometry, with the benefit of easy adaptation with geometrically based processing algorithms. These full reference metrics compare the distorted and original 3D models to compute the quality score. For example, Karni and Gotsman [17] proposed combining the RMS geometric distance between corresponding vertices with the RMS distance of their Laplacian coordinates (which reflect a degree of smoothness of the surface). Lavoué [18] and Torkhani et al. [19] proposed metrics based on local differences of curvature statistics, while Vasa and Rus [20] considered the dihedral angle differences. These metrics consider local variations of attribute values at vertex or edge level, which are then pooled into a global score. In contrast, Corsini et al. [21] and Wang et al. [22] compute global roughness values per model and then derive a simple global roughness difference. For the roughness calculation, they consider respectively dihedral angles and variance of the geometric Laplacian [21] and Laplacian of the Gaussian curvature [22]. Some authors also proposed quality assessment metrics for textured 3D mesh [23], [24], which integrate both texture and geometry information. Similar to the image quality metrics, some of these latter algorithms [19], [20], [22] integrate perceptually motivated mechanisms such as visual masking.

However the HVS is complex in terms of 3D data visualization, involving a large number of screen-based, model-based and perception-based parameters. Hence, inferring HVS characteristics such as masking and contrast sensitivity, from the 3D geometry is challenging. Instead, we propose to treat the HVS as a black box from which we wish to learn the input (curvatures, dihedral angles, geometric Laplacian, curvature Laplacian and geometric distances) and output (data quality) relationship. In this work, we use two Mean Opinion Score (MOS) datasets to learn which attributes are relevant to predict visual quality. We demonstrate that an optimal combination of perceptually relevant attributes, determined through machine learning, can produce better results than the current state-of-the-art mesh quality metrics. The idea of using subjective data and machine learning techniques to train a predictor is growing up in different areas of computer graphics. For instance, it has been recently used by Secord et al. [25] and Herzog et al. [14] who address completely different research questions: the best viewpoint selection of a 3D object [25] and the detection of local rendering artefacts in 2D images [14].

\section{Perceptually RELEVANT ATtRibutes}

As reviewed in the previous section, different attributes have been used for 3D Mesh Visual Quality assessment (MVQ), curvature computations [18], dihedral angles [20], Geometric Laplacian [17], and Laplacian of Gaussian curvature [22]. It is worth noting that most of existing MVQ metrics, except those working on textured meshes, focus on using individual attributes separately. We hypothesize that a combination of these attributes can deliver better results (as validated in Section V). In this section we highlight eight geometric attributes, denoted below by $a_{1}$ to $a_{8}$, which are commonly used in MVQ metrics. Our objective is to evaluate their respective efficiencies in predicting visual distortions and to propose a more effective metric which is determined using machine learning techniques.

- $a_{1}$ : Minimum curvature.

- $a_{2}$ : Maximum curvature.

- $a_{3}$ : Mean curvature.

- $a_{4}$ : Gaussian curvature - To ensure the stability of the four curvature fields, following the solution adopted in [18], we evaluate the curvature tensor using the method from [26] on a large neighborhood around each vertex, a geodesic disk approximated by the intersection of the surface with a sphere centered at the vertex.

- $a_{5}$ : Dihedral angle [20] - It is computed for each edge and corresponds to the angle between the normals of its adjacent faces. The computed values are then averaged for each vertex.

- $a_{6}$ : Geometric Laplacian [17] [27] - Given a vertex $v$ (i.e. a $3 \mathrm{D}$ vector), it is defined as:

$$
G L(v)=v-\frac{\sum_{i \in n(v)}\left(l_{i}^{-1} v_{i}\right)}{\sum_{i \in n(v)} l_{i}^{-1}}
$$


where $v_{i}$ are neighbors of $v, n(v)$ is the set of indices of the neighbors of $v$, and $l_{i}$ is the Euclidean distance from $v$ to $v_{i}$.

- $a_{7}$ : Laplacian of Gaussian curvature [22] - It corresponds to the Laplacian operator applied on the Gaussian curvature field. Note that in [22], the authors use the cotangent weights discretization of the Laplace operator while we consider the simpler graph Laplacian.

- $a_{8}$ : The 3D geometric position.

$a_{6}$ and $a_{8}$ are $3 \mathrm{D}$ vectors, while the others are scalar values.

Each attribute $a_{i}$ computed locally for each vertex $j$ of a mesh is denoted by $a_{i}^{j}$. We want to evaluate the individual performance of each attribute in predicting the visual distortion using a full reference scenario. Therefore, given a distorted model $\mathcal{D}$ and a reference model $\mathcal{R}$, the local per-vertex distortion of each attribute is computed as $\Delta a_{i}^{j}=\left\|\mathcal{D}\left(a_{i}^{j}\right)-\mathcal{R}\left(a_{i}^{j}\right)\right\|$, where $\mathcal{D}\left(a_{i}^{j}\right)$ (resp. $\mathcal{R}\left(a_{i}^{j}\right)$ ) is the value of the attribute $a_{i}$ for the $j^{\text {th }}$ vertex of mesh $\mathcal{D}$ (resp. $\mathcal{R}$ ). The distributions of these local distortions are relevant for our objective of visual quality prediction. Figure 1 illustrates a reference model (top left) and a distorted model (top right) obtained after smoothing. The computed local distortion distributions using geometric positions $\Delta a_{8}^{j}$ and mean curvature $\Delta a_{3}^{j}$ are illustrated in the second and third row of Figure 1.

Following the above process can use statistics, mean, variance, skewness and kurtosis, extracted from these distortion distributions as visual quality predictors. For illustration purpose in this paper, we consider the mean of the distribution:

$$
\Delta a_{i}=\frac{1}{n} \sum_{j=0}^{n} \Delta a_{i}^{j}
$$

as the quality predictor for a given attribute $a_{i}$, denoted by $\Delta a_{i}$ and named attribute metric in the rest of the paper. Note that $n$ is the number of vertices in $\mathcal{R}$ and $\mathcal{D}$, which are supposed to share the same connectivity and thus same number of vertices. This connectivity constraint can easily be relaxed by projecting the vertices from $\mathcal{D}$ on $\mathcal{R}$, as explained in [18].

\section{Mean Opinion Score Databases}

For each attribute $a_{i}$ presented above, in order to assess the correlation between the prediction outcome of an attribute metric $\Delta a_{i}$ and the average human opinion, we need to establish subjective opinions on the 3D models. In this regard, we deploy two databases that we created earlier which contain different categories of distorted and reference objects, as well as the corresponding mean opinion scores reflecting their qualities collected from human subjects. These two databases were made available to the public (http://liris.cnrs.fr/guillaume.lavoue/data/datasets.html) and have been used in many 3D mesh quality assessment related
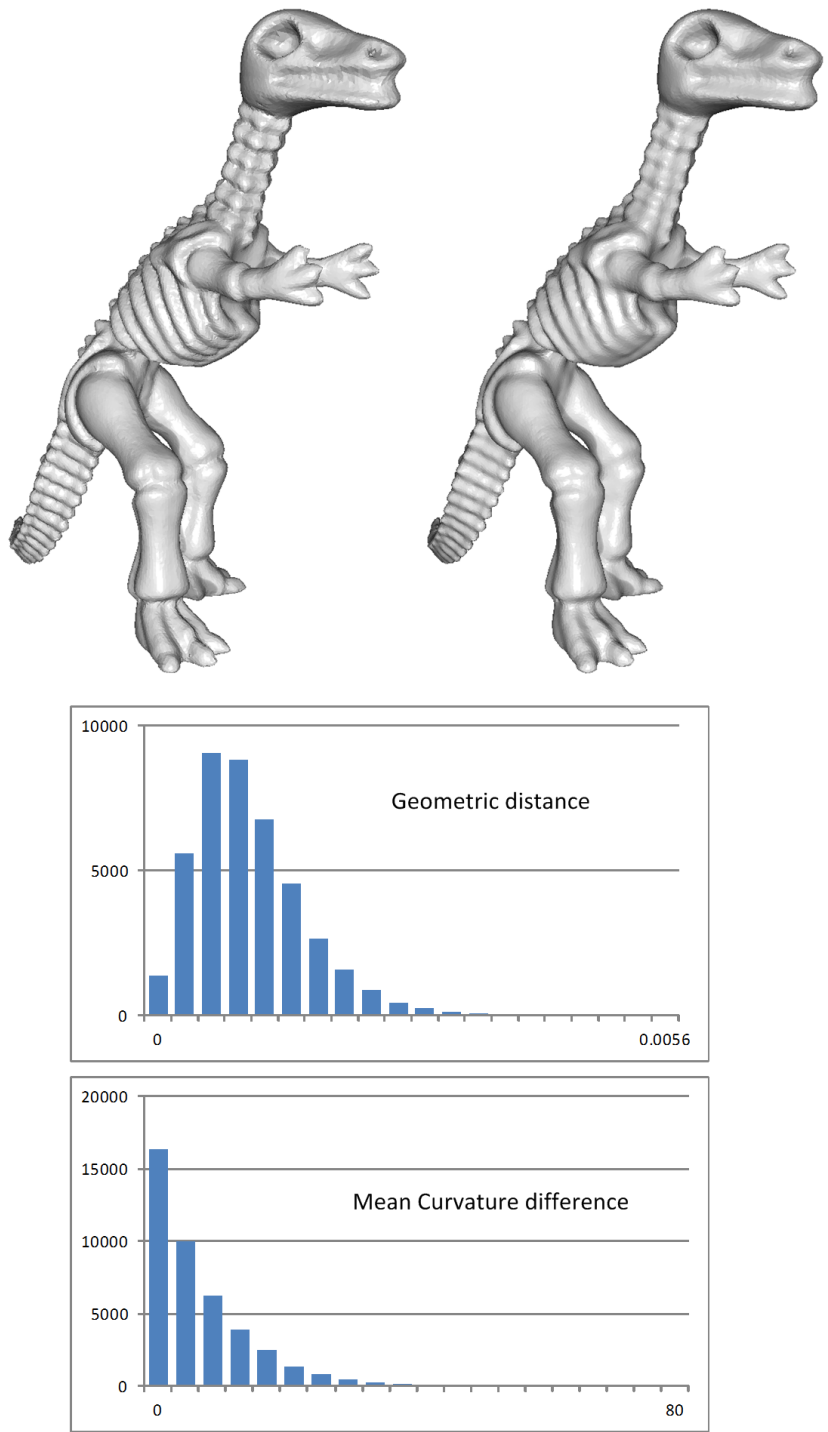

Fig. 1. Top row: a reference model (left) and a distorted version after smoothing (right). Middle row: distribution of the per-vertex geometric distances. Bottom row: distribution of the per-vertex mean curvature differences.

studies [1], [2], [18]-[20], [22]. These MOS databases are described below and illustrated in Figure 2.

The LIRIS/EPFL General-Purpose Database [28] was created at the EPFL, Switzerland. It contains 88 models having between $40 \mathrm{~K}$ and $50 \mathrm{~K}$ vertices generated from 4 reference objects (Armadillo, Dyno, Venus and RockerArm). Two types of distortions (noise addition and smoothing) are applied with different strengths and at four categories of surface locations: uniformly on the whole object, on smooth areas, on rough areas and on intermediate areas (i.e. between smooth and rough). 12 observers participated in the user study. 3D objects illustrated in Figures 1 and 2 (top row) come from this database. 
The LIRIS Masking Database [29] was created at the Université of Lyon, France. It contains 26 models having between $9 \mathrm{~K}$ and $40 \mathrm{~K}$ vertices generated from 4 reference objects (Armadillo, Bimba, Dyno and Lion) specifically chosen because they contain significantly smooth and rough areas. The only distortion is noise addition using three strengths applied on either smooth or rough regions. The purpose of using this database is to evaluate the responses of the metrics to visual masking effects. 11 observers participated in this user study. As expected, the added noise is less visible on rough compared to smooth regions. Hence, given the same distortion level MVQ metrics should predict higher visual impact on smooth surfaces. Objects from this database are illustrated in Figure 2 (bottom row).

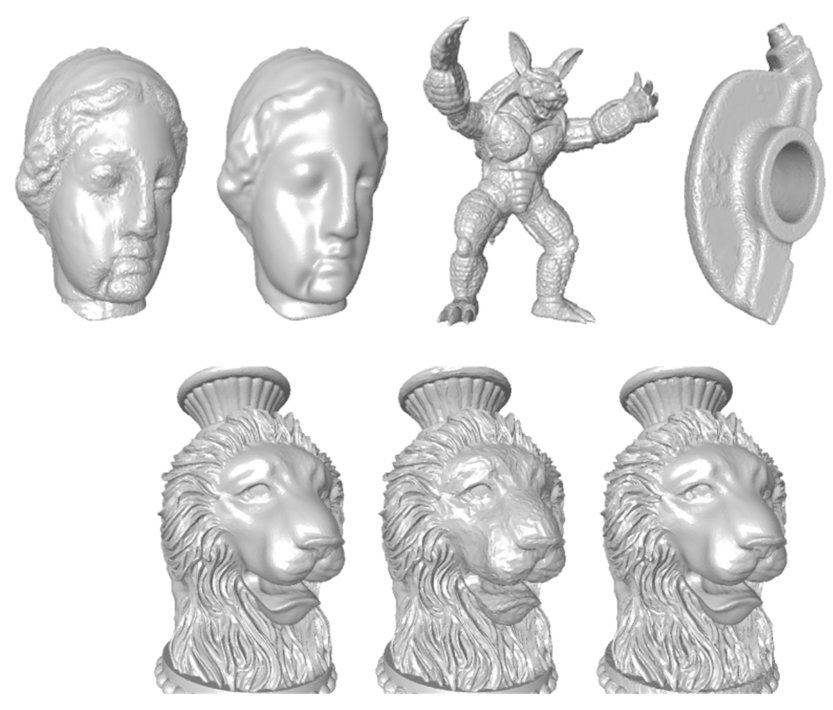

Fig. 2. Top row: Models from the LIRIS/EPFL General-Purpose Database. Noise and smoothing are applied in different areas. bottom row: Models from the LIRIS Masking Database. Noise is applied either on smooth or rough areas.

\section{Attribute Learning ANd Evaluation}

\section{A. Single and multi-attribute prediction performance}

To evaluate the prediction performance of each attribute, we use the LIRIS/EPFL General-Purpose database described above, which contains 88 models associated with mean opinion scores. Figure 3 illustrates correlation of each of the 8 attribute metrics $\Delta a_{i}$ with the MOS. In this experiment, we compute for each attribute, a quality predictor $P_{i}$ using a simple linear model: $P_{i}=\omega_{0}+\omega_{i} . \Delta a_{i}$. The weights $\omega_{0}, \omega_{i}$ are obtained through linear regression for each attribute. Table I illustrates the correlation of the corresponding predicted values with the MOS. For the fitting, the weights are learned on a random subset of half of the models and tested on the other half. The root mean square errors of the predictions are also provided for comparison.

We can see from Figure 3 and Table I that the best features for visual quality prediction are the curvature (mean and

\begin{tabular}{lcc}
\hline Attribute & Correlation & RMS \\
\hline$\Delta a_{1}$ Min Curvature & 59.9 & 1.60 \\
$\Delta a_{2}$ Max Curvature & $\mathbf{6 9 . 0}$ & $\mathbf{1 . 4 3}$ \\
$\Delta a_{3}$ Mean Curvature & $\mathbf{7 0 . 3}$ & $\mathbf{1 . 4 0}$ \\
$\Delta a_{4}$ Gauss Curvature & 52.9 & 1.70 \\
$\Delta a_{5}$ Dihedral Angle & $\mathbf{7 0 . 8}$ & $\mathbf{1 . 4 4}$ \\
$\Delta a_{6}$ Geometric Laplacian & 31.8 & 1.89 \\
$\Delta a_{7}$ Laplacian of Gaussian Curv. & 48.8 & 1.74 \\
$\Delta a_{8}$ Geometric position & 34.4 & 1.89 \\
Linear combination & $\mathbf{8 3 . 9}$ & $\mathbf{1 . 0 4}$ \\
\hline
\end{tabular}

TABLE I

PREDICTION ACCURACY, IN TERMS OF PEARSON CORRELATION AND ROOT MEAN SQUARE ERROR, OF THE INDIVIDUAL ATTRIBUTES AND THEIR COMBINATION REGARDING SUBJECTIVE MEAN OPINION SCORES, AFTER LINEAR REGRESSION.

maximum) and the dihedral angle. They each demonstrate around $70 \%$ of Pearson correlation with the MOS. As reported in recent studies [1], [2], the geometric difference attribute is shown to be a poor predictor ( $34 \%$ correlation). These results suggest that mean and maximum curvatures, and dihedral angle are good candidates for modeling mesh visual quality metrics. In general, the Laplacian-based attributes $\left(a_{6}, a_{7}\right)$ [17] [22] give poor results.

We hypothesize that a combination of these attributes should considerably improve the prediction performance. To validate this hypothesis, we start by considering a linear combination of the eight attributes as a new predictor $P_{i}=\omega_{0}+\sum_{i=1}^{8} \omega_{i} . \Delta a_{i}$. The weights $\omega_{i}$ are learned by multi-linear regression based on the Akaike information criterion [30], trained on a random subset of half of the models and tested on the other half. The obtained weights are presented in Table II. We observe that the resulting quality prediction model is very accurate, as shown in the last row of Table I. This linear combination model has $83.9 \%$ Pearson correlation with the MOS, and a low RMS. This encouraging result shows that an appropriate combination of the attributes can considerably improve the prediction efficiency.

\begin{tabular}{ccccccccc}
\hline$\omega_{0}$ & $\omega_{1}$ & $\omega_{2}$ & $\omega_{3}$ & $\omega_{4}$ & $\omega_{5}$ & $\omega_{6}$ & $\omega_{7}$ & $\omega_{8}$ \\
\hline 3.71 & -0.80 & -1.00 & 2.09 & $-4.7 \mathrm{E}-3$ & 0 & $-7.46 \mathrm{E} 3$ & $5.8 \mathrm{E}-3$ & $8.27 \mathrm{E} 3$ \\
\hline
\end{tabular}

TABLE II

WEIGHTS FOR OUR MULTI-ATTRIBUTE PREDICTION MODEL. ALL ATTRIBUTES ARE SCALED APPROPRIATELY.

To further validate the robustness of our proposed metric, we select the top-performing ones based on the comparison results above and test them using the LIRIS Masking Database, after the training step has been completed using the GeneralPurpose database. Note that the distortions generated in these two databases are quite different. This can result in a weak metric because the knowledge obtained from the training set may not consitute a good representation of the testing set. Table III reports the prediction results which clearly demonstrate that single attribute predictors are not efficient in this experiment. However, our proposed metric continues to have high correla- 
$\Delta a_{1}$ Min. Curvature

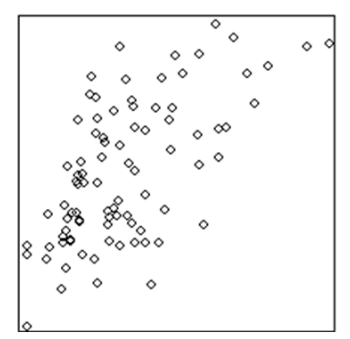

$\Delta a_{5}$ Dihedral Angle

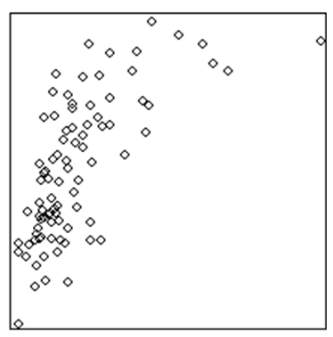

$\Delta a_{2}$ Max. Curvature

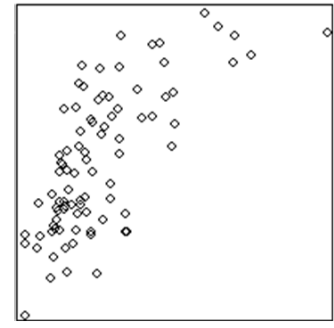

$\Delta a_{6}$ Geo. Laplacian

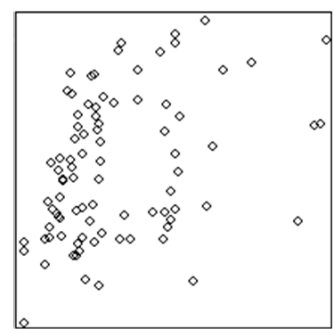

$\Delta a_{3}$ Mean Curvature

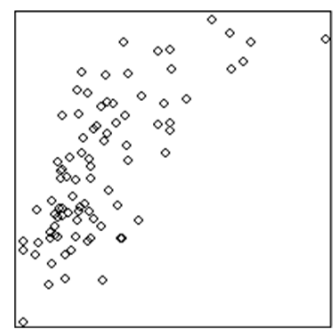

$\Delta a_{7}$ Gauss. Laplac.

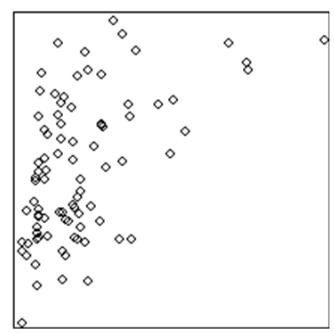

$\Delta a_{4}$ Gauss Curvature

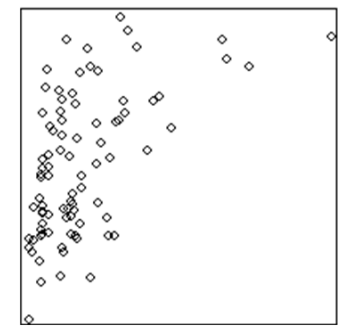

$\Delta a_{8}$ Geom. Position

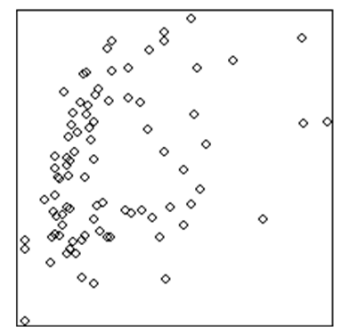

Fig. 3. Plots of the different attribute metrics $\Delta a_{i}$ ( $X$ axis) versus the mean opinion scores ( $Y$ axis).

tion with mean opinion scores.

\begin{tabular}{lcc}
\hline Attribute & Correlation & RMS \\
\hline$\Delta a_{2}$ Max Curvature & 38.1 & 3.53 \\
$\Delta a_{3}$ Mean Curvature & 42.9 & 3.44 \\
$\Delta a_{5}$ Dihedral Angle & 22.0 & 3.92 \\
Linear combination & $\mathbf{8 5 . 7}$ & $\mathbf{3 . 5 8}$ \\
\hline
\end{tabular}

TABLE III

PREDICTION ACCURACY FOR THE MASKING DATABASE, AFTER TRAINING THE LINEAR MODELS ON THE GENERAL-PURPOSE DATABASE.

\section{B. Linear combination of attributes as a new quality metric}

We have seen in the previous section that our multi-attribute model has high correlation with mean opinion scores from both databases. In this section, we compare its efficiency with both simple (Hausdorff, RMS) and advanced metrics (MSDM2 [18], FMPD [22] and DAME [20]).

To evaluate the performance of a quality metric, the correlation is computed between the mean opinion scores of the objects and their associated metrics' values. Two correlation coefficients are commonly used: the Spearman rank order correlation which measures the monotonic association between the MOS and the metric values, and the Pearson linear correlation which measures the prediction accuracy. Before computing these values, we perform a psychometric curve fitting, to optimize the matching between the values given by the objective metrics and the subjective scores provided by human subjects. This step takes into account the saturation effect associated with human senses. For this reason, psychometric curves exhibit a typical sigmoid shape that penalizes the strongest stimuli. This fitting is used here to evaluate the performance of the perceptually-based measures, but could also be included in the perceptual metrics for specific applications [21], [28]. In this work, we apply the Gaussian psychometric function to every tested metrics $P$ :

$$
P_{\text {fit }}=g(a, b, P)=\frac{1}{2 \pi} \int_{a+b P}^{\infty} e^{-\frac{t^{2}}{2}} d t
$$

where $a$ and $b$ are the parameters estimated by nonlinear leastsquares data fitting.

\begin{tabular}{lcccc}
\hline & \multicolumn{2}{c}{ General Purpose } & \multicolumn{2}{c}{ Masking } \\
& Spearman & Pearson & Spearman & Pearson \\
\hline Hausdorff & 13.8 & 11.4 & 26.6 & 20.2 \\
RMS & 26.8 & 28.1 & 48.8 & 41.2 \\
MSDM2 [18] & 80.4 & 81.4 & 89.6 & 87.3 \\
FMPD [22] & 81.9 & 83.5 & 80.2 & 80.8 \\
DAME [20] & 76.6 & 75.2 & 68.1 & 58.6 \\
Linear comb. & $\mathbf{8 9 . 9}$ & $\mathbf{9 0 . 3}$ & $\mathbf{8 6 . 0}$ & $\mathbf{8 5 . 7}$ \\
\hline
\end{tabular}

TABLE IV

Spearman and Pearson correlation (\%) Between Mean Opinion SCORES AND VALUES FROM THE METRICS, AFTER PSYCHOMETRIC CURVE FITTING.

Table IV present the comparison results. Observe that our multi-attribute model significantly outperforms the other metrics in the General-Purpose database and (together with the MSDM2) delivers the best results in the Masking database. Simplicity is an advantage of our scheme, compared with metrics integrating complex masking filters (FMPD, DAME) and costly local neighborhood calculation (MSDM2). Using our metric, the average computation time needed for the comparison of two objects with 50k vertices is 1 second on a $2 \mathrm{GHz}$ intel i7 CPU. The speed is similar for the FMPD and DAME metrics, but our results are significantly better. MSDM2 is much slower (20 seconds on average). Figure 4 and Figure 5 illustrate the psychometric curve fitting between the values of our multi-attribute predictor and the subjective scores over the General-purpose and Masking databases. Each data point represents a distorted model. 


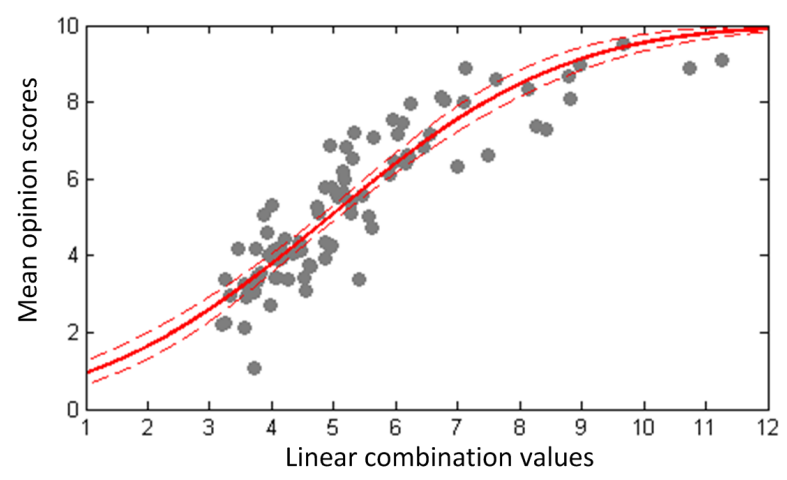

Fig. 4. Subjective MOS vs metric values for the General-Purpose database. Each circle represents a distorted model. The cumulative Gaussian fitted curve is displayed in red.

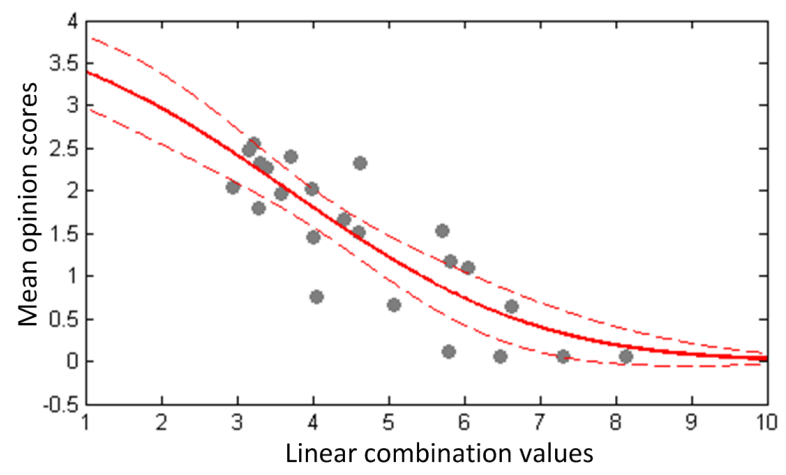

Fig. 5. Subjective MOS vs metric values for the Masking database. Each circle represents a distorted model. The cumulative Gaussian fitted curve is displayed in red.

\section{CONCLUSION AND PERSPECTIVES}

We have presented quantitative evaluations for several 3D mesh geometric attributes with regard to their efficiency in predicting the perceived distortion in 3D models. We have also proposed a new quality metric based on an optimized combination of these attributes, using machine learning techniques on two databases created based on subjective opinions. The proposed visual quality metric is cost effective and easy to implement but provides good prediction results. Moreover, it can easily accommodate new mesh surface attributes, and thus is an integral component of a larger computational model. In future work, we will enhance our metric by comparing the simple averaging of local distortion distributions with more perceptually-related $p$-norms as well as other descriptors of local distributions such as variance, skewness and kurtosis.

\section{REFERENCES}

[1] G. Lavoué and M. Corsini, "A comparison of perceptually-based metrics for objective evaluation of geometry processing," IEEE Transactions on Multimedia, vol. 12, no. 7, pp. 636-649, 2010.

[2] M. Corsini, M. C. Larabi, G. Lavoué, O. Petík, L. Váša, and K. Wang, "Perceptual Metrics for Static and Dynamic Triangle Meshes," Computer Graphics Forum, vol. 32, no. 1, pp. 101-125, Feb. 2013.

[3] Z. Wang and A. Bovik, "Modern image quality assessment," Synthesis Lectures on Image, Video, and Multimedia Processing, vol. 2, no. 1, pp. $1-156,2006$.
[4] A. McNamara, K. Mania, M. Banks, and C. Healey, "Perceptuallymotivated graphics, visualization and 3D displays," ACM Siggraph Courses, 2010.

[5] Z. Wang, A. Bovik, H. Sheikh, and E. Simoncelli, "Image quality assessment: From error visibility to structural similarity," IEEE Transactions on Image Processing, vol. 13, no. 4, pp. 600-612, 2004.

[6] J. Lubin, "The use of psychophysical data and models in the analysis of display system performance," in Digital Images and Human Vision, A. B. Watson, Ed., Oct. 1993, pp. 163-178.

[7] S. Daly, "The visible differences predictor: an algorithm for the assessment of image fidelity," in Digital images and human vision, Andrew B. Watson, Ed. Cambridge: MIT Press, Oct. 1993, pp. 179-206.

[8] P. Lindstrom, "Out-of-core simplification of large polygonal models," in ACM Siggraph, 2000, pp. 259-262.

[9] L. Qu and G. Meyer, "Perceptually guided polygon reduction," IEEE Transactions on Visualization and Computer Graphics, vol. 14, no. 5, pp. 1015-1029, 2008.

[10] M. Reddy, "Perceptually optimized 3D graphics," IEEE Computer Graphics and Applications, vol. 21, no. 5, pp. 68-75, 2001.

[11] M. Bolin and G. Meyer, "A perceptually based adaptive sampling algorithm," in ACM Siggraph, 1998, pp. 299-309.

[12] M. Cadík, R. Herzog, and R. Mantiuk, "New measurements reveal weaknesses of image quality metrics in evaluating graphics artifacts," ACM Siggraph, 2012.

[13] T. O. Aydin, M. Čadík, K. Myszkowski, and H.-P. Seidel, "Video quality assessment for computer graphics applications," ACM Transactions on Graphics, vol. 29, no. 6, p. 1, Dec. 2010.

[14] R. Herzog, M. Cadik, T. O. Aydin, K. I. Kim, K. Myszkowski, and H.p. Seidel, "NoRM : No-Reference Image Quality Metric for Realistic Image Synthesis," Computer Graphics Forum, vol. 31, no. 2, 2012.

[15] R. Mantiuk, "Quantifying image quality in graphics: perspective on subjective and objective metrics and their performance," IS\&T/SPIE Electronic Imaging, pp. 86510K-86510K-13, Mar. 2013.

[16] B. E. Rogowitz and H. Rushmeier, "Are image quality metrics adequate to evaluate the quality of geometric objects?" Proceedings of SPIE, pp. 340-348, 2001.

[17] Z. Karni and C. Gotsman, "Spectral compression of mesh geometry," in ACM Siggraph, 2000, pp. 279-286.

[18] G. Lavoué, "A Multiscale Metric for 3D Mesh Visual Quality Assessment," Computer Graphics Forum, vol. 30, no. 5, pp. 1427-1437, 2011.

[19] F. Torkhani, K. Wang, and J.-m. Chassery, "A Curvature Tensor Distance for Mesh Visual Quality Assessment," in International Conference on Computer Vision and Graphics, 2012.

[20] L. Váša and J. Rus, "Dihedral Angle Mesh Error: a fast perception correlated distortion measure for fixed connectivity triangle meshes," Computer Graphics Forum, vol. 31, no. 5, 2012.

[21] M. Corsini, E. D. Gelasca, T. Ebrahimi, and M. Barni, "Watermarked 3D Mesh Quality Assessment," IEEE Transactions on Multimedia, vol. 9, no. 2, pp. 247-256, Feb. 2007.

[22] K. Wang, F. Torkhani, and A. Montanvert, "A Fast Roughness-Based Approach to the Assessment of 3D Mesh Visual Quality," Computers \& Graphics, 2012.

[23] D. Tian and G. AlRegib, "FQM: A Fast Quality Measure for Efficient Transmission of Textured 3D Models," in ACM Multimedia, 2004, pp. 684-691.

[24] I. Cheng and P. Boulanger, "A Visual Quality Prediction Model for 3D Texture," Proc. EUROGRAPHICS, pp. 101-104, 2005.

[25] A. Secord, J. Lu, A. Finkelstein, and M. Singh, "Perceptual models of viewpoint preference," in ACM Siggraph, 2011.

[26] D. Cohen-Steiner and J. Morvan, "Restricted delaunay triangulations and normal cycle," in 19th Annu. ACM Sympos. Comput. Geom., 2003.

[27] O. Sorkine, D. Cohen-Or, and S. Toldeo, "High-pass quantization for mesh encoding," in Eurographics Symposium on Geometry Processing, 2003, pp. 42-51.

[28] G. Lavoue, E. Drelie Gelasca, F. Dupont, A. Baskurt, and T. Ebrahimi, "Perceptually driven 3D distance metrics with application to watermarking," in SPIE, vol. 6312, no. 1. SPIE, Aug. 2006, pp. 63 120L-63 120L12.

[29] G. Lavoué, "A local roughness measure for 3D meshes and its application to visual masking," ACM Transactions on Applied Perception (TAP), vol. 5 , no. $4,2009$.

[30] H. Akaike, "Information theory as an extension of the maximum likelihood principle," in Second International Symposium on Information Theory, 1973, pp. $267-281$. 